\title{
Apoptosis-promoting properties of miR-3074-5p in MC3T3-E1 cells under iron overload conditions
}

\author{
Yi Feng ${ }^{1,3 \dagger}$, Pei-Yan He ${ }^{2 \dagger}$, Wei-Dong Kong ${ }^{1,3}$, Wan-Jing Cen ${ }^{1,3}$, Peng-Lin Wang ${ }^{1,3}$, Chang Liu 1,3 , Wu Zhang ${ }^{1,3}$, \\ Shu-Shu $\mathrm{Li}^{1,3^{*}}$ and Jian-Wei Jiang ${ }^{2^{*}}$
}

${ }^{*}$ Correspondence:
lishushu1987@126.com;
jjw703@jnu.edu.cn
${ }^{\dagger}$ Yi Feng and Pei-Yan He
contributed equally to this
study
${ }^{1}$ Department
of Orthodontics, The
First Affiliated Hospital
of Jinan University, No.613
Huangpu Road West,
Guangzhou 510630, China
${ }^{2}$ Department
of Biochemistry, Basic
Medical College, Jinan
University, No.601
Huangpu Road West,
Guangzhou 510632, China
Full list of author information
is available at the end of the
article

*Correspondence: lishushu1987@126.com;

${ }^{+}$Yi Feng and Pei-Yan He contributed equally to this

of Jinan University, No.613

Huangpu Road West,

Guangzhou 510630, China

${ }^{2}$ Department

of Biochemistry, Basic

Medical College, Jinan

University, No.601

Guangzhou 510632, China

is available at the end of the article

\begin{abstract}
Background: Iron overload can promote the development of osteoporosis by inducing apoptosis in osteoblasts. However, the mechanism by which miRNAs regulate apoptosis in osteoblasts under iron overload has not been elucidated.

Method: The miRNA expression profile in MC3T3-E1 cells under iron overload was detected by next generation sequencing. QRT-PCR was used to determine the expression of miR-3074-5p in MC3T3-E1 cells under iron overload. The proliferation of MC3T3-E1 cells was tested using CCK-8 assays, and apoptosis was measured using flow cytometry. The miRanda and TargetScan databases were used to predict the target genes of miR-3074-5p. Interaction between miR-3074-5p and the potential target gene was validated by qRT-PCR, luciferase reporter assay and western blotting.

Results: We found that iron overload decreased the cell viability and induced apoptosis of MC3T3-E1 cells. The results of next generation sequencing analysis showed that miR-3074-5p expression was significantly increased in MC3T3-E1 cells under iron overload conditions, which was confirmed by further experiments. The inhibition of miR-3074-5p attenuated the apoptosis of iron-overloaded MC3T3-E1 cells. Furthermore, the expression of Smad4 was decreased and was inversely correlated with miR-3074-5p expression, and overexpression of Smad4 partially reversed the viability inhibition of iron-overloaded MC3T3-E1 cells by relieving the suppression of ERK, AKT, and Stat3 phosphorylation, suggesting its regulatory role in the viability inhibition of iron-overloaded MC3T3-E1 cells. The luciferase reporter assay results showed that Smad4 was the target gene of miR-3074-5p.
\end{abstract}

Conclusion: miR-3074-5p functions as an apoptosis promoter in iron-overloaded MC3T3-E1 cells by directly targeting Smad4.

Keywords: Iron overload, Osteoporosis, Apoptosis, MC3T3-E1 cells, miRNA-3074-5p

\section{Background}

Iron is one of the essential nutrients for mammals. However, iron accumulation in tissues, which is characteristic of the elderly, is a risk factor for infection, osteoporosis, and several chronic diseases [1]. Osteoporosis is a systemic disease that causes reduced

(c) The Author(s), 2021. Open Access This article is licensed under a Creative Commons Attribution 4.0 International License, which permits use, sharing, adaptation, distribution and reproduction in any medium or format, as long as you give appropriate credit to the original author(s) and the source, provide a link to the Creative Commons licence, and indicate if changes were made. The images or other third party material in this article are included in the article's Creative Commons licence, unless indicated otherwise in a credit line to the material. If material is not included in the article's Creative Commons licence and your intended use is not permitted by statutory regulation or exceeds the permitted use, you will need to obtain permission directly from the copyright holder. To view a copy of this licence, visit http:// creativecommons.org/licenses/by/4.0/. 
bone strength and an increased risk of fracture, which is caused by reduced bone mass and bone microstructure destruction. Fracturing is a serious consequence of osteoporosis, which can significantly increase the morbidity and mortality of patients, and cause considerable family and socioeconomic burdens [2]. Evidence from several studies has demonstrated that iron overload directly inhibits bone remodeling [3-5]. In a study of fifty patients with osteoporosis, $88 \%$ of them were iron overloaded, suggesting that iron overload may play a role in promoting the development and progression of osteoporosis [6]. The steady state of the skeletal system depends on the dynamic equilibrium between osteoblast-mediated osteogenesis and osteoclast-mediated bone destruction in bone metabolism. When the number or function of osteoblasts decreased, bone formation was less than resorption, resulting in loss of alveolar bone mass. Many in vivo and in vitro studies have suggested that iron overload could reduce bone formation and increase bone resorption, which is associated with pathological bone loss [7-9]. Cell studies in vitro have found that iron overload induced osteoblast apoptosis by increasing intracellular reactive oxygen species and activating the mitochondrial apoptosis pathway [10-12].

miRNAs are a class of endogenous non-coding single-stranded small RNAs with a length of approximately 20-22 nucleotides. These RNAs play a key role in cell metabolism, proliferation, autophagy, apoptosis, differentiation and other biological processes, and their expression changes are related to many human diseases [13-15]. Studies have shown that miRNAs participate in regulating apoptosis. Several apoptosis-related genes are regulated by miRNAs [16-18]. miR-1 promoted nitric oxide-induced apoptosis of MC3T3-E1 cells by targeting HSP-70 [19]. miR-214 alleviated apoptosis of $\mathrm{H}_{2} \mathrm{O}_{2}$-treated MC3T3-E1 cells by suppressing oxidative stress [20]. However, the mechanism by which miRNAs regulate apoptosis in MC3T3-E1 cells under iron overload has not been reported.

The present study used an iron overload model of MC3T3-E1 cells induced by a high concentration of ferric ammonium citrate (FAC) to simulate an environment of iron overload and analyzed the miRNA expression profiles in MC3T3-E1 cells under iron overload conditions. The results showed that increased miR-3074-5p due to iron overload caused apoptosis of MC3T3-E1 cells. Overexpression of miR-3074-5p inhibited Smad4 in MC3T3-E1 cells. Restoration of Smad4 rescued the effects of miR-3074-5p in FAC-treated MC3T3-E1 cells. The present data show the apoptosis-promoting properties of miR-3074-5p by regulating its downstream gene target Smad4 in MC3T3-E1 cells under iron overload in vitro.

\section{Methods}

\section{Cell culture, drug treatment, and transfection}

The murine preosteoblast cell line MC3T3-E1 was obtained from American Type Culture Collection (Manassas, VA, USA) and stored at the Department of Biochemistry, Medical College of Jinan University (Guangzhou, China). MC3T3-E1 cells were cultured in $\alpha$-MEM (Gibco-BRL, Gaithersburg, MD, USA) containing $10 \%$ fetal bovine serum at $37{ }^{\circ} \mathrm{C}$ in an incubator with $5 \%$ carbon dioxide [21]. To simulate an in vitro environment of iron overload (iron overload conditions), cells were treated with $0,0.6,1.2,1.8,2.4$ and $3.0 \mathrm{mM}$ ferric ammonium citrate (FAC; Sangon, Shanghai, China). After exposure 
to FAC for specified times, all samples were collected for the following experiments. To overexpress and block miR-3074-5p in MC3T3-E1 cells, cells were transfected with miR-3074-5p mimic, inhibitor, and the negative control (Ribo, Guangzhou, China) separately at a working concentration of $100 \mathrm{nM}$ using the Ribo FECT CP Transfection Kit (Ribo, Guangzhou, China) following the manufacturer's guidelines. miRNA inhibitor is a chemically synthesized 21-23 nt oligonucleotide modified by 2'-methoxy, which can specifically bind to mature miRNA to reduce its regulatory effect in cells. The sequences of mimic, mimic NC, inhibitor and inhibitor NC used are shown in Table 1. To overexpress Smad4 in MC3T3-E1 cells, the cells were transfected with pcDNA3.1-Smad4 and a pcDNA3.1-negative control using Lipofectamine 2000 (Invitrogen, Carlsbad, CA, USA). Intracellular expression of Smad4 was confirmed by western blot analysis.

\section{Cell viability measurement}

Cell viability was detected using a CCK-8 assay (Sigma-Aldrich, St Louis, USA). MC3T3-E1 cells were cultured in 96-well plates at $3 \times 10^{3}$ cells per well. After 24,48 and $72 \mathrm{~h}$ of FAC treatment, the cell inhibition rate was determined with a CCK- 8 assay following the manufacturer's guidelines [22]. Cell inhibition rate $=(1-$ mean of OD values of the treatment group/mean of OD values of the control group) $\times 100 \%$.

\section{Hoechst staining to detect apoptosis-related morphologic changes}

MC3T3-E1 cells were cultured in 6-well plates at $5 \times 10^{4}$ cells per well. After $24 \mathrm{~h}$ of 0 and $1.8 \mathrm{mM}$ FAC treatment for $24 \mathrm{~h}$, the cells were fixed. A Hoechst 33,258 staining kit (Beyotime, Shanghai, China) was used for cell staining [23]. Observation of apoptotic cells was carried out with a fluorescence microscope (Olympus, Tokyo, Japan) at a $350 \mathrm{~nm}$ excitation wavelength and $460 \mathrm{~nm}$ emission wavelength.

\section{Annexin V-FITC/PI staining to determine apoptosis}

Apoptosis of MC3T3-E1 cells was determined using an Annexin V-FITC/PI Kit (Beyotime, Shanghai, China). In brief, cells were seeded into 6 -well plates at $2.0 \times 10^{4}$ cells/well. After treatment with 0, 0.6, 1.2, 1.8 and $2.4 \mathrm{mM} \mathrm{FAC} \mathrm{for} 24 \mathrm{~h}$, the cells were collected, incubated with Annexin V-FITC and PI following the manufacturer's guidelines [24], and finally analyzed on a FACSCalibur flow cytometer (Becton Dickinson Immunocytometry Systems, San Jose, CA, USA).

Table 1 Sequences of mimic, mimic NC, inhibitor, and inhibitor NC

\begin{tabular}{ll}
\hline mimic/inhibitor/NC & Sequences \\
\hline miR-3074-5p mimic & 5'-GUUCCUGCUGAACUGAGCCAGUUGGCU \\
mimic NC & CAGUUCAGCAGGAACUU-3' \\
miR-3074-5p inhibitor & Sense: 5'-UUCUCCGAACGUGUCACGUTT-3' \\
Inhibitor NC & Antisense: 5'-ACGUGACACGUUCGGAGAATT-3' \\
& 5'-ACUGGCUCAGUUCAGCAGGAAC-3' \\
& 5'-CAGUACUUUUGUGUAGUACAA-3' \\
\hline
\end{tabular}




\section{Western blotting}

The total protein in cells was extracted and the concentration was assessed. Western blotting was conducted as described previously [25]. Antibodies against caspase 3, cleaved caspase-3, XIAP, c-IAP2, survivin, AKT, p-AKT, ERK, p-ERK, Stat3, p-Stat3, Smad4, and GAPDH were all obtained from Cell Signaling Technology Co., Ltd. (Danvers, MA, USA).

\section{miRNA profiling}

The total RNA was isolated from MC3T3-E1 cells treated with or without 1.8 mM FAC for $24 \mathrm{~h}$. miRNA next-generation sequencing analysis was carried out by BGI Genomics Co., Ltd (Shenzhen, China). Different expression of miRNAs between MC3T3-E1 cells treated without FAC (control group) and with FAC (FAC group) was identified through the $\log 2$ Ratio (FAC/Control), which was calculated as the $\log 2$ value of the fold change, with threshold sets for up- and downregulated genes of a log2Ratio (FAC/Control) $\geq 1.0$ or $\leq 1.0$ with a $P$ value $<0.05$.

\section{qRT-PCR}

The total RNA was isolated using TRIzol reagent (Keygen, Jiangsu, China). cDNA was synthesized from mRNA with the cDNA Synthesis SuperMix (Bimake, Houston, TX, USA) and from miRNA with a Reverse Transcription Kit (Ribo, Guangzhou, China). qPCR was performed to detect the expression levels of miR-3074-5p and Smad4 using the Bulge-Loop miRNA qRT-PCR Starter Kit (Ribo, Guangzhou, China) and $2 \times$ SYBR Green qPCR Master Mix (Bimake, Houston, TX, USA) [26], respectively. The specific primers used are shown in Table 2. U6 and GAPDH were used as internal controls of miRNA and mRNA, respectively.

\section{Bioinformatics analysis}

To understand the function of differentially expressed miRNAs in MC3T3 cells treated with or without FAC, KEGG pathway analysis was conducted to perform pathway enrichment analysis. In this study, enrichment factors of KEGG pathways in the top 20 were chosen and shown. Two bioinformatics software programs, TargetScan and miRanda, were used for target gene prediction. The intersection of their prediction results was taken as the final result.

Table 2 Primer sequences for qRT-PCR

\begin{tabular}{ll}
\hline Gene & Sequences \\
\hline miR-3074-5p & Forward: 5'-GCGGTTCCTGCTGAACTGA-3' \\
Smad4 & Reverse: 5'-AGTGCAGGGTCCGAGGTATT-3' \\
& Forward: 5'-AGTTCACAATGAGCTTGCATTC-3' \\
U6 & Reverse: 5'-TTCAAAGTAAGCAATGGAGCAC-3' \\
& Forward: 5'-TGCAGAGGATCTAATT-3' \\
GAPDH & Reverse: 5'-GAAAGACCAGTCCAAGTCC-3' \\
& Forward: 5'-TGTGTCCGTCGATCTGA-3' \\
& Reverse: 5'-TTGCTGTTTGTGAAGTCGCAGGAG-3'
\end{tabular}




\section{Dual-luciferase report gene assay}

A wide-type $3^{\prime}$-UTR fragment of Smad4 mRNA (5'...CUGUCAUGAGUGGAGCAG GAAG...3') containing a putative miR-3074-5p-binding site (position 2954-2960) was cloned into the psiCHECK ${ }_{2}$ vector, named WT-Smad4-3'-UTR luciferase vector. The mutation of the $3^{\prime}$-UTR fragment of Smad4 mRNA ( $5^{\prime}$...CUGUCAUGAGUGGAG CUCCAAG...3') was constructed using the QuickChange mutagenesis kit (Agilent Technologies, California, USA) and cloned into the $\mathrm{psiCHECK}_{2}$ vector to construct the MUT-Smad4-3'-UTR luciferase vector [22]. HEK293T cells were transfected with psiCHECK2 reporter plasmids containing either the wild-type or mutant type of the 3 UTR of Smad4 in the presence of miR-3074-5p mimic and NC using Lipofectamine 2000, and cultured for $48 \mathrm{~h}$ before being harvested for experiments and analysis. The relative luciferase activity was implemented using the Dual-Luciferase Reporter Assay Kit (Yeasen, Shanghai, China).

\section{Statistical analysis}

Quantitative data were expressed as the mean \pm SD. SPSS 17.0 software (SPSS, Chicago, IL, USA) and GraphPad Prism 7.0 (GraphPad, San Diego, CA, USA) were used for statistical analysis. Independent samples $t$ tests (unpaired $t$-tests) were performed for comparisons between two groups. One-way ANOVA was performed for comparisons among groups. $P<0.05$ was considered to indicate statistical significance.

\section{Results}

Iron overload decreased the viability and induced apoptosis of MC3T3-E1 cells

After exposing MC3T3-E1 cells to FAC $(0,0.6,1.2,1.8,2.4$ and $3.0 \mathrm{mM})$ separately for 24, 48 and $72 \mathrm{~h}$, the CCK- 8 assay revealed that FAC at each concentration inhibited the proliferation of MC3T3-E1 cells in a concentration- and time-dependent manner, compared with the untreated control group $(P<0.05)$ (Fig. 1a). To observe the morphological changes of FAC-treated MC3T3-E1 cells, cells treated with 0 and $1.8 \mathrm{mM}$ FAC separately for $24 \mathrm{~h}$ were stained with a Hoechst 33,258 Staining Kit. The results of Hoechst staining showed that the nuclei of apoptotic MC3T3-E1 cells treated with 1.8 mM FAC were densely stained, which was the typical morphological change of apoptotic cells (Fig. 1b, c). To assess whether iron overload mediated cytotoxicity in MC3T3-E1 cells was related to apoptosis, Annexin V-FITC/PI staining was used to detect the apoptosis rate of MC3T3-E1 cells. Flow cytometry analysis showed that the late apoptosis rate increased from 1.63 to $61.85 \%$ as FAC concentrations increased from $0 \mathrm{mM}$ to $2.4 \mathrm{mM}$, suggesting a dose-dependent effect of FAC on apoptosis in MC3T3-E1 cells (Fig. 1d). To confirm that iron overload did induce apoptosis of MC3T3-E1 cells at the protein level, the expression of apoptosis-related proteins in MC3T3-E1 cells was evaluated by western blot analysis. In this study, dose-dependent downregulation of XIAP, c-IAP2, survivin, p-AKT, p-ERK and p-Stat3, as well as upregulation of cleaved-caspase 3, was observed in MC3T3-E1 cells (0.6-1.8 mM FAC) for $24 \mathrm{~h}$ (Fig. 1e). All of the results demonstrate that iron overload promotes apoptosis of MC3T3-E1 cells. 


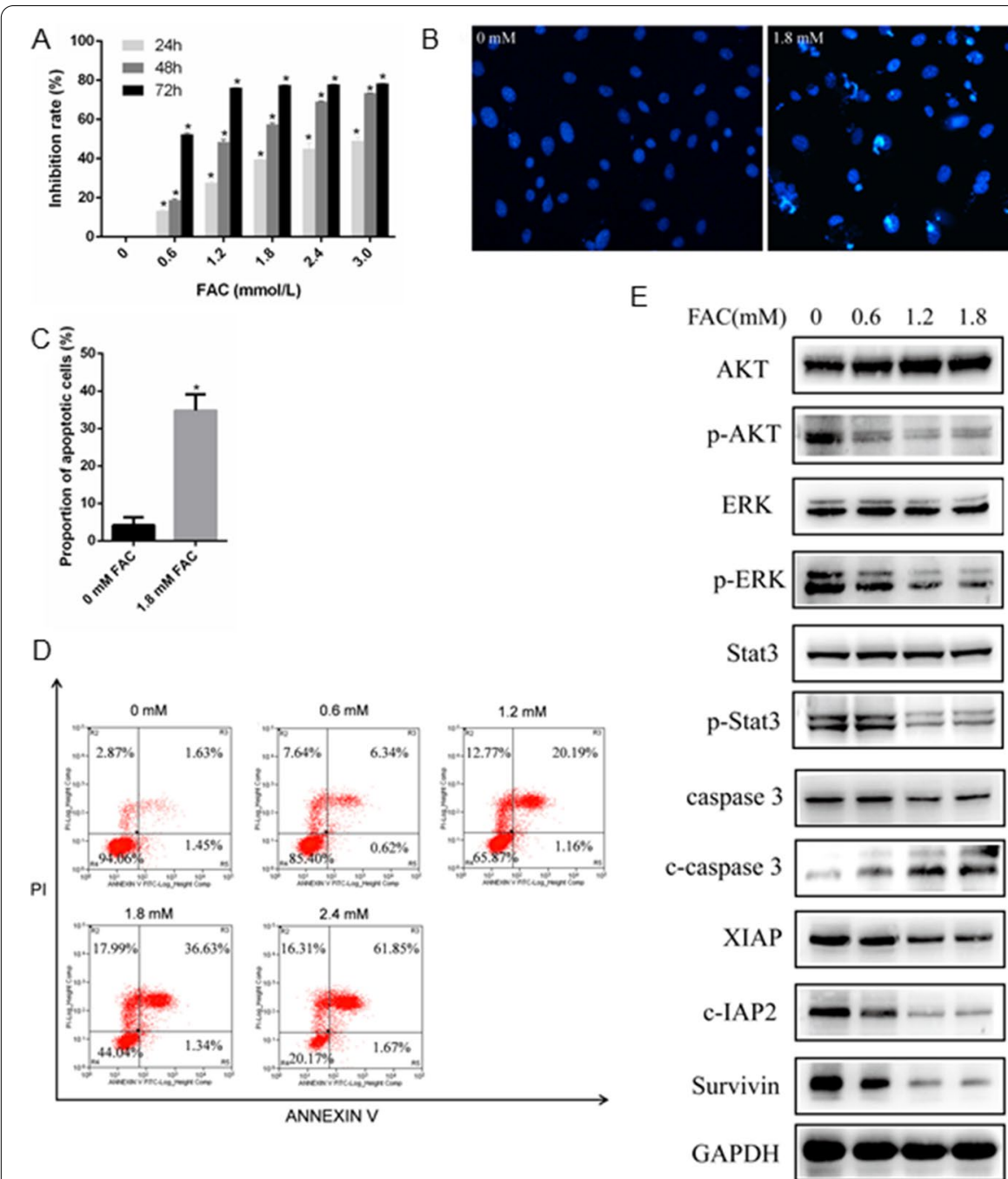

Fig. 1 Iron overload decreased the viability and increased the apoptosis rate of MC3T3-E1 cells. A The viability of MC3T3-E1 cells was evaluated by CCK-8 assay after exposure to FAC (0-3.0 mM) for 24, 48 and $72 \mathrm{~h} .{ }^{*} P<0.05$ vs. the control. B MC3T3-E1 cells were treated with 0 and $1.8 \mathrm{mM}$ FAC for $24 \mathrm{~h}$. The nuclei of apoptotic cells treated with $1.8 \mathrm{mM} \mathrm{FAC}$ were densely stained with Hoechst 33,258, and were condensed and bright. Scale bars: $100 \mu \mathrm{m}$. C Quantitative analysis of apoptosis based on Hoechst 33,258 staining. ${ }^{*} P<0.05$ vs. the control. D Cells were exposed to FAC (0-2.4 mM) for $24 \mathrm{~h}$. Apoptotic MC3T3-E1 cells stained with Annexin $\mathrm{V} / \mathrm{PI}$ were determined by flow cytometric analysis. $\mathbf{E}$ Apoptosis-related protein expression levels of cells were evaluated by western blot analysis after exposure to $0-1.8 \mathrm{mM} \mathrm{FAC}$ for $24 \mathrm{~h}$

\section{Iron overload induced miR-3074-5p expression in MC3T3-E1 cells}

Differentially expressed miRNAs between MC3T3-E1 cells treated with and without $1.8 \mathrm{mM}$ FAC for $24 \mathrm{~h}$ were identified via miRNA next-generation sequencing analysis (Fig. 2a). The raw data from the analysis have been deposited into the NCBI SRA database under the accession code PRJNA506880. We focused on miR-3074-5p, which displayed significant upregulation in iron-overloaded MC3T3-E1 cells, compared with the control group $(P<0.05)$. In order to validate this result, we examined the expression profile of miR-3074-5p in MC3T3-E1 cells treated with and without $1.8 \mathrm{mM}$ FAC for $24 \mathrm{~h}$. The qRT-PCR results showed that miR-3074-5p expression significantly increased in MC3T3-E1 cells exposed to FAC, compared to the untreated control group $(P<0.05)$ 

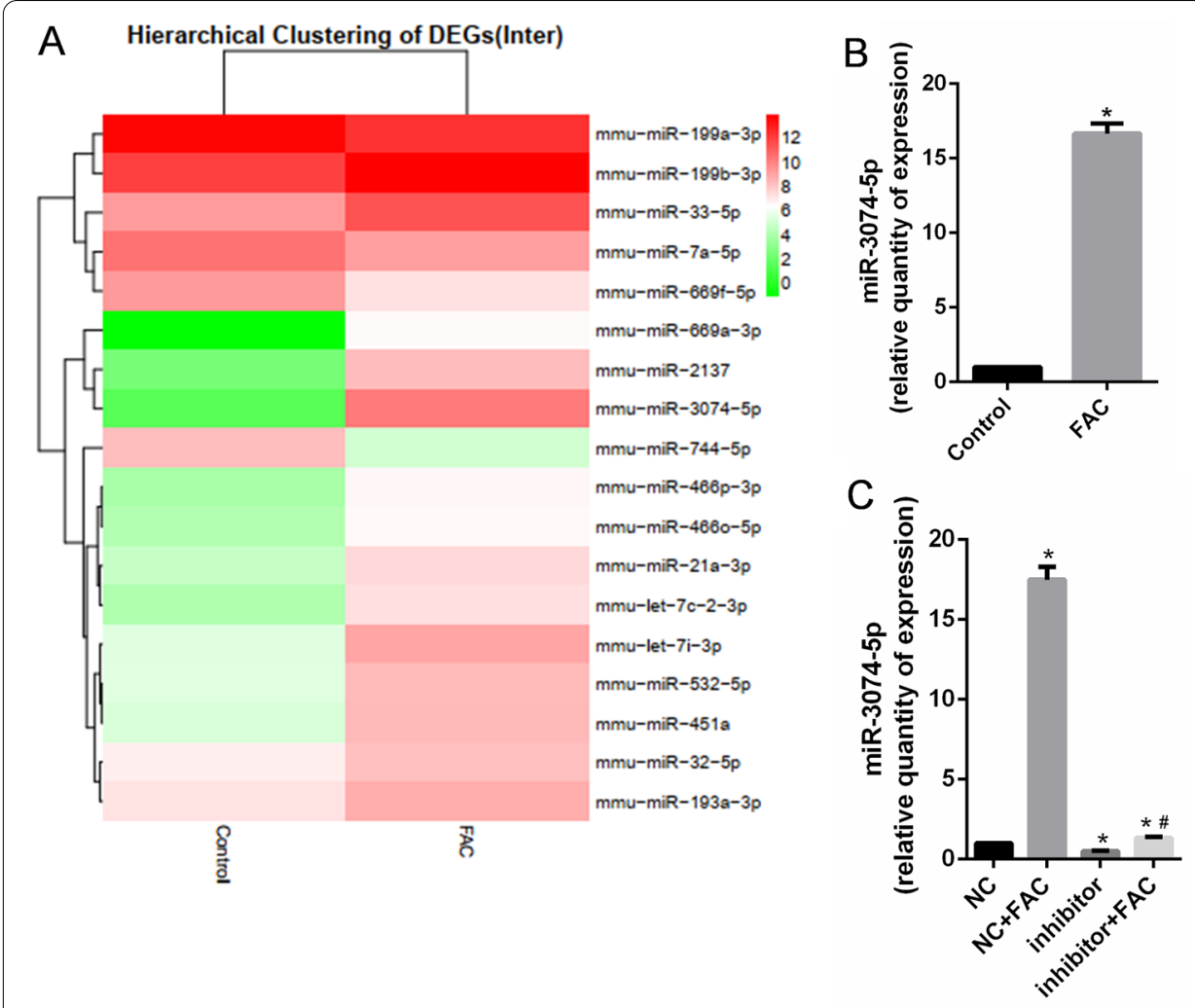

Fig. 2 miR-3074-5p expression was increased in MC3T3-E1 cells under iron overload conditions. A Heatmap of 18 miRNAs displaying significant upregulation (red pixels) or downregulation (green pixels) in MC3T3-E1 cells treated with $1.8 \mathrm{mM}$ FAC, compared with the control. B, C miR-3074-5p expression was validated by qRT-PCR. Inhibitor indicated miR-3074-5p inhibitor. NC indicates the negative control. ${ }^{*} P<0.05$ vs. the NC group. ${ }^{P} P<0.05$ vs. the NC + FAC group

(Fig. 2b). We also found that cells transfected with miR-3074-5p inhibitor could rescue FAC-induced miR-3074-5p upregulation in MC3T3-E1 cells (Fig. 2c). The above results suggest a potential function of miR-3074-5p in MC3T3-E1 cells under iron overload.

\section{miR-3074-5p mediated iron overload-induced apoptosis of MC3T3-E1 cells}

To explore the biological impact of miR-3074-5p on the apoptosis of MC3T3-E1 cells under iron overload conditions, gain- and loss-of-function experiments were performed by transfecting cells with a miR-3074-5p mimic or inhibitor, respectively. The CCK-8 assay was employed to define the effects of miR-3074-5p on cell viability and Annexin V-FITC/PI staining was performed to confirm the function of miR3074-5p in cell apoptosis. The results revealed a significant increase in the inhibition rate of miR-3074-5p mimic-treated MC3T3-E1 cells under iron overload conditions (mimic + FAC group) compared with the negative control (NC)-treated MC3T3E1 cells in the iron overload group (NC + FAC group) and the NC only group $(P<0.05)$. There was a significant decrease in the inhibition rate of miR-3074-5p inhibitor-treated MC3T3-E1 cells under iron overload (inhibitor + FAC group) compared with the negative control (NC)-treated MC3T3-E1 cells under iron overload $(\mathrm{NC}+\mathrm{FAC}$ group) $(P<0.05)$ (Fig. 3a). Consistently, the apoptosis rate was increased 


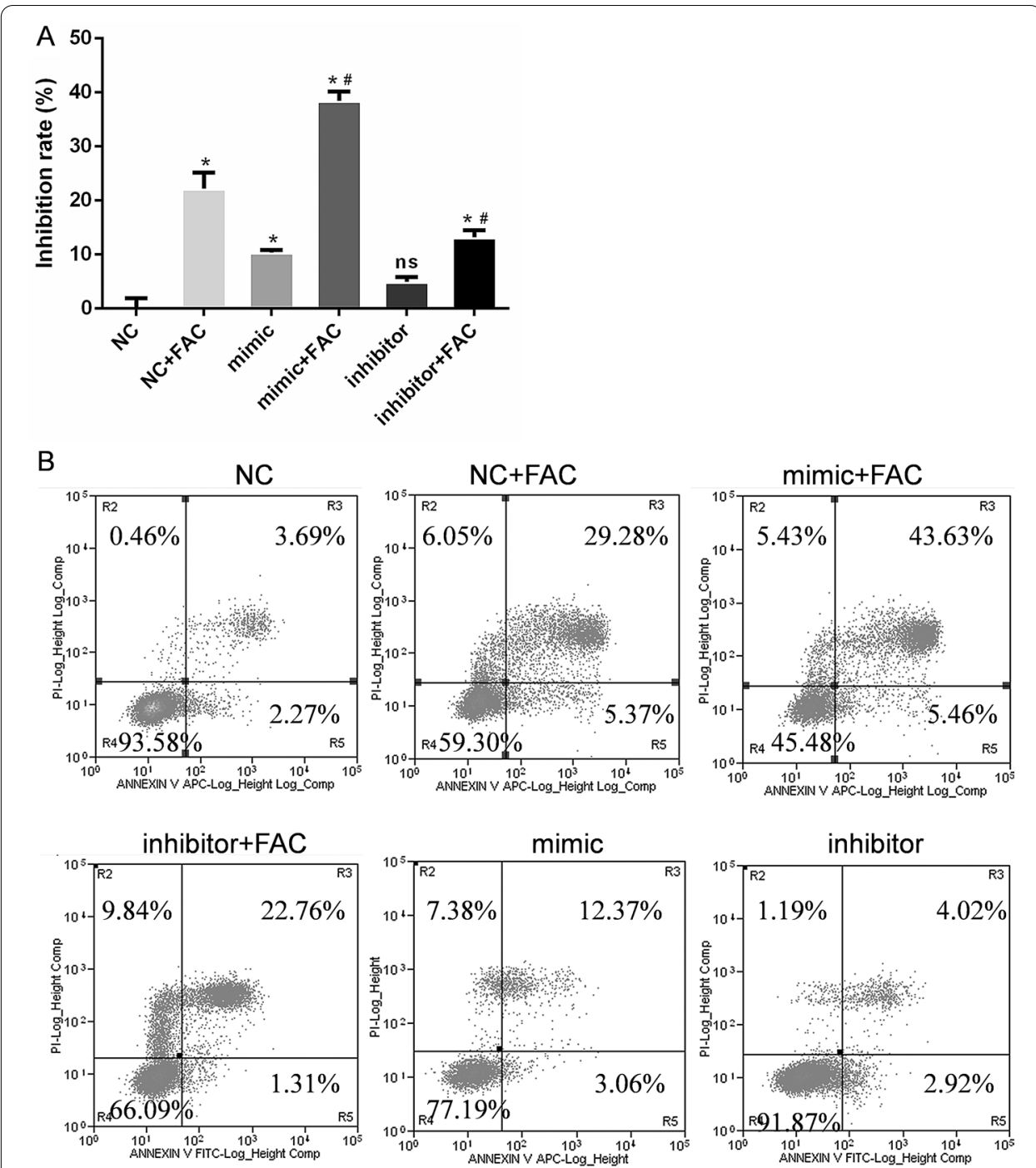

Fig. 3 miR-3074-5p mediated the apoptosis of MC3T3-E1 cells under iron overload conditions. A Gain- and loss-of-function experiments were performed by transfecting cells with a miR-3074-5p mimic and miR-3074-5p inhibitor, respectively. The CCK-8 assay was used to investigate the effect of miR-3074-5p on cell viability. ${ }^{*} P<0.05$ vs. the NC. ${ }^{\#} P<0.05$ vs. the NC + FAC group. ns $P>0.05$ vs. the NC group. FAC concentration: $1.8 \mathrm{mM}$. B Annexin V-FITC/PI staining was performed to confirm the function of miR-3074-5p in cell apoptosis. C Quantitative analysis of late apoptosis rate based on Annexin V-FITC/PI staining. ${ }^{*} P<0.05$ vs. the NC. ${ }^{\#} P<0.05$ vs. the NC + FAC group. ns $P>0.05$ vs. the NC group

in MC3T3-E1 cells transfected with the miR-3074-5p mimic under iron overload (mimic + FAC group) compared with the negative control (NC)-treated MC3T3-E1 cells in the iron overload group (NC + FAC group) and the NC only group $(P<0.05)$. Conversely, the apoptosis rate was decreased in cells that were transfected with the miR-3074-5p inhibitor under iron overload (inhibitor + FAC group), compared with the negative control (NC)-treated MC3T3-E1 cells under iron overload (NC+FAC group) $(P<0.05)$ (Fig. 3b). These results clearly indicate that aberrant expression of miR-3074-5p contributes to apoptosis of iron-overloaded MC3T3-E1 cells. 


\section{Results of bioinformatic analysis}

KEGG pathway analysis showed the target mRNAs of the differentially expressed miRNAs in MC3T3 cells treated with or without FAC. The top 20 enriched pathways with $P<0.05$ were chosen and are shown (Additional file 1: Fig. S1a). Notably, these target genes of the differentially expressed miRNAs were enriched in two cellular survival- and death-related pathways, the FoxO signaling pathway and the mTOR signaling pathway (Additional file 1: Fig. S1a). Target mRNAs of miR-3074-5p were predicted with the miRanda and TargetScan databases. Eight hundred and sixty-four targets were identified by TargetScan and 450 targets were identified by miRanda; 314 targets overlapped between the two database. Among the predicted target genes, Smad4 was related to the FoxO signaling pathway and Jmjd8 was related to the mTOR signaling pathway (Additional file 1: Fig. S1b).

\section{miR-3074-5p targeted Smad4 in MC3T3-E1 cells}

Further experiments are required to explore the molecular mechanisms linking miR3074-5p with apoptosis of iron-overloaded MC3T3-E1 cells. Smad4 was identified as a potential target mRNA of miR-3074-5p. According to the results of bioinformatics analysis, miR-3074-5p might bind to 3UTR of Smad4 (Fig. 4a). In this study, the expression of Smad4 in MC3T3-E1 cells under iron overload was lower than that in the untreated control group $(P<0.05)$ (Fig. $4 \mathrm{~b}-\mathrm{d})$. The expression of Smad4 mRNA and protein in MC3T3-E1 cells under iron overload was further reduced by overexpression of miR3074-5p achieved by transfecting cells with a miR-3074-5p mimic, and the expression of Smad4 mRNA and protein in MC3T3-E1 cells under iron overload was rescued by inhibition of miR-3074-5p by transfecting cells with a miR-3074-5p inhibitor (Fig. 4b-d), indicating that miR-3074-5p may mediate Smad4 mRNA degradation by 3'UTR targeting. Moreover, miR-3074-5p regulates iron overload-promoted apoptosis in MC3T3E1 cells by targeting Smad4, which was further confirmed by a dual-luciferase reporter assay (Fig. 4e).

\section{Smad4 attenuated miR-3074-5p mediated apoptosis in MC3T3-E1 cells induced by iron overload}

Gain- and loss-of-function experiments were performed by transfecting cells with pcDNA3.1-Smad4 and miR-3074-5p mimic, respectively. The CCK-8 assay was employed to determine the effects of Smad4 on cell viability. The results revealed a significant increase in the inhibition rate of pcDNA3.1-NC+miR-3074-5p mimic-treated MC3T3-E1 cells (pcDNA3.1-NC + mimic group) compared with that of pcDNA3.1-NC-treated MC3T3E1 cells (pcDNA3.1-NC group) $(P<0.05)$ (Fig. $5 \mathrm{a})$. The inhibition rate of pcDNA3.1Smad4+ miR-3074-5p mimic-treated MC3T3-E1 cells (pcDNA3.1-Smad4+ mimic group) was significantly lower than that of pcDNA3.1-NC + miR-3074-5p mimic-treated MC3T3E1 cells (pcDNA3.1-NC + mimic group) $(P<0.05)$ (Fig. 5a). Consistently, the expression of pAKT, pERK, p-Stat3, and Smad4 was lower in MC3T3-E1 cells transfected with the pcDNA3.1-NC+miR-3074-5p mimic (pcDNA3.1-NC+mimic group), compared with the pcDNA3.1-NC-treated MC3T3-E1 cells (pcDNA3.1-NC group). Conversely, the expression of pAKT, pERK, p-Stat3, and Smad4 was higher in cells transfected with the 


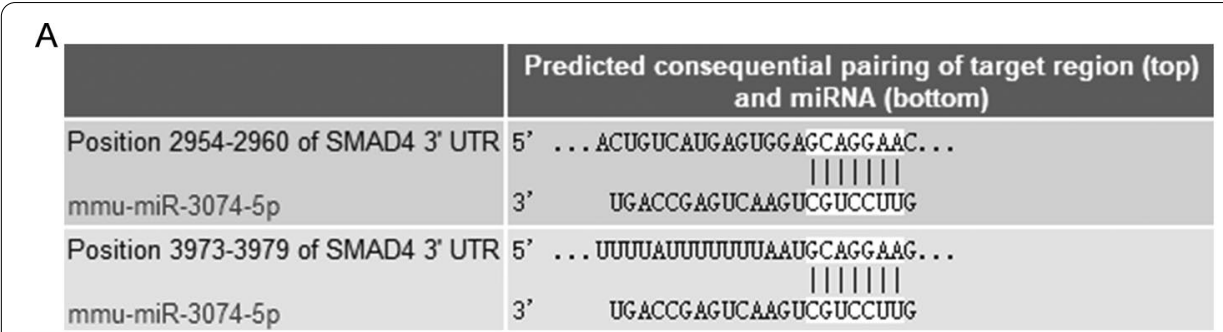

$\mathrm{B}$

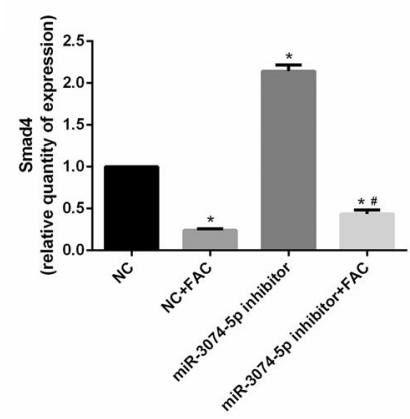

D

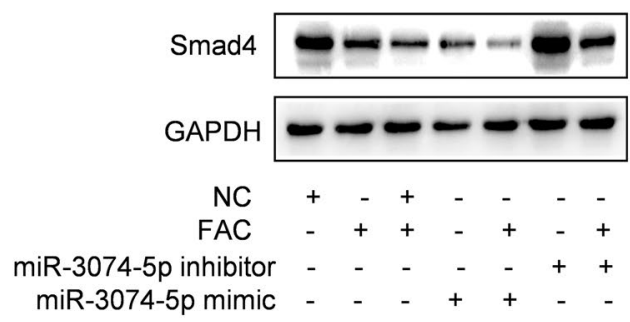

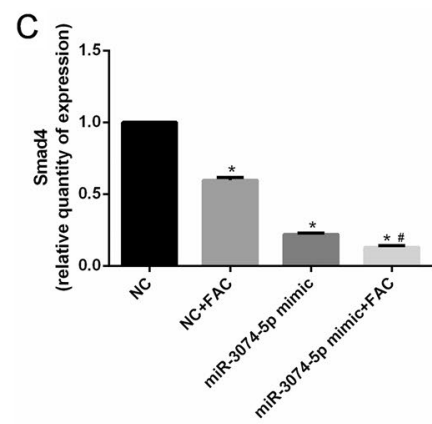

$E$

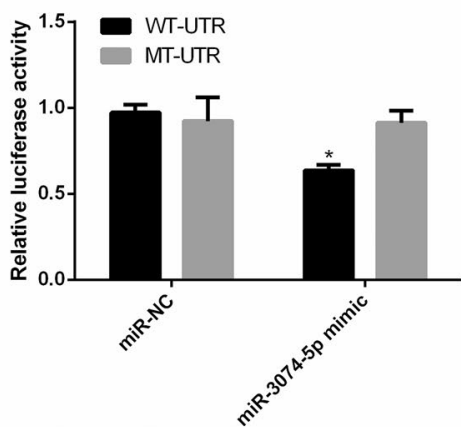

Fig. 4 Smad4 mRNA and protein were decreased in MC3T3-E1 cells under iron overload and were inversely correlated with miR-3074-5p expression. A Bioinformatics analysis showed that miR-3074-5p might bind to 3'UTR region of Smad4. B MC3T3-E1 cells were transfected with the miR-3074-5p inhibitor and NC separately and treated with or without $1.8 \mathrm{mM}$ FAC. Smad4 mRNA was quantified by qRT-PCR. C Cells were transfected with the miR-3074-5p mimic and NC separately and treated with or without $1.8 \mathrm{mM} \mathrm{FAC.} \mathrm{Smad4} \mathrm{mRNA} \mathrm{was}$ quantified by qRT-PCR. ${ }^{*} P<0.05$ vs. the NC group. ${ }^{\#} P<0.05$ vs. the NC+ FAC group. D MC3T3-E1 cells were transfected with miR-3074-5p mimic, miR-3074-5p inhibitor and negative control separately and treated with or without $1.8 \mathrm{mM}$ FAC. Smad4 protein was detected by qRT-PCR. E The dual-luciferase report assay demonstrated that miR-3074-5p directly targets the 3'UTR of Smad4. The miR-3074-5p mimic repressed the activity of the wild-type (WT) Smad4 3'-UTR ( $5^{\prime}$... CUGUCAUGAGUGGAGCAGGAAG ...3), but not that of the mutant type (MT) Smad4 3'-UTR (5'...CUGUCAUGAGUGGAGCUCCAAG ...3). * $P<0.05$ vs. the NC group

pcDNA3.1-Smad4+miR-3074-5p mimic (pcDNA3.1-Smad4+mimic group) compared with the pcDNA3.1-NC+ miR-3074-5p mimic-treated MC3T3-E1 cells (pcDNA3.1$\mathrm{NC}+$ mimic group) (Fig. 5b). These results indicate that restoration of Smad4 can rescue the viability inhibition of MC3T3-E1 cells mediated by miR-3074-5p under iron overload.

\section{Discussion}

Iron is a necessary auxiliary factor in many biological processes of the human body. Normally, iron metabolism is in a stable state in the human body. In osteoporosis patients, the internal environment of the bone marrow is in an iron overload state. Iron 


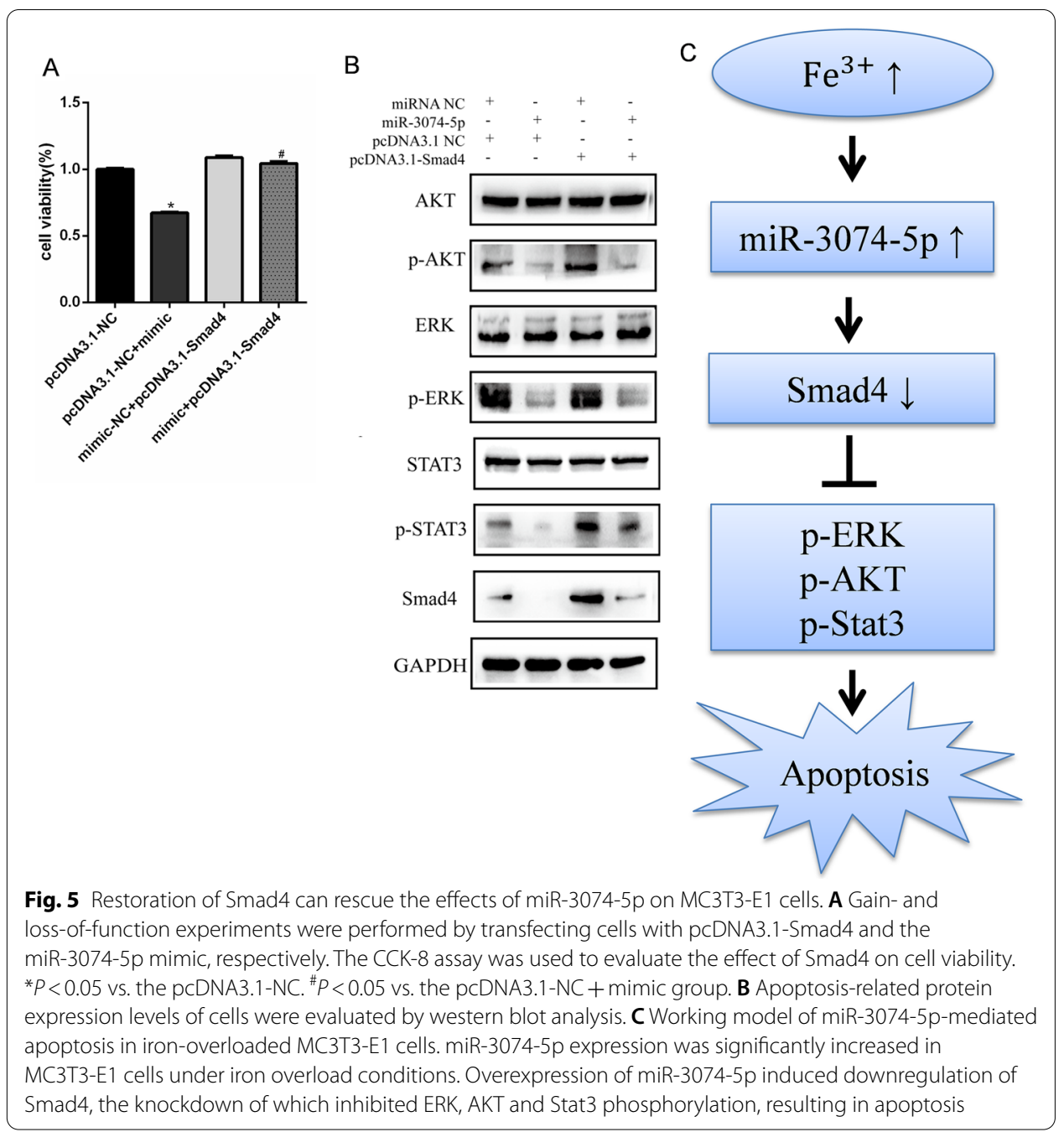

overload-induced osteoblast proliferation inhibition and osteoblast apoptosis are averse to bone remodeling in osteoporosis patients. In vivo and in vitro studies have shown that iron overload inhibits osteoblast proliferation and promotes osteoblast apoptosis. Iron overload induces osteoblast apoptosis by increasing ROS and activating the mitochondrial apoptosis pathway $[10,11]$. However, the mechanism of activating the apoptosis pathway by targeting apoptosis-related genes via upstream microRNAs is still unclear. The purpose of this study was to analyze the miRNA expression profiles and evaluate the function of miR-3074-5p in the apoptosis of MC3T3-E1 cells under iron overload conditions and the potential mechanisms.

It has been reported that a high concentration of FAC could cause apoptosis of MC3T3-E1 cells $[10,25]$. The results of this study showed that FAC at concentrations above $0.6 \mathrm{mM}$ could induce apoptosis of MC3T3-E1 cells. Caspase 3 is known as one of the effectors of apoptosis. Endogenous, exogenous and endoplasmic reticulum pathways of apoptosis can ultimately activate caspase 3 through different signaling pathways and lead to apoptosis. In this study, the expression of caspase 3 decreased and that of cleaved-caspase 3 increased in FAC-treated MC3T3-E1 cells, suggesting that caspase 3 
was activated and induced apoptosis. The IAP family is an inhibitor of apoptosis in cells, and includes XIAP, c-IAP1, c-IAP2, survivin, and NAIP. Among these proteins, c-IAP1 and 2 can inhibit the activation of caspase 9 by interfering with the Apaf-1/cytochrome C complex, thereby inhibiting the activation of caspase 3, while XIAP can directly inhibit the activation of caspase 3 [27]. Survivin can directly inhibit the activation of caspase 3 and caspase 9 [28]. AKT, ERK, and Stat3 play important roles in apoptosis and cell survival. They can inhibit apoptosis and promote cell survival by phosphorylation of themselves and inactivation of their downstream antiapoptotic targets. Apoptosis occurs when the phosphorylation of AKT, ERK, and Stat3 is blocked by stimulating factors [2931]. In this study, the expression levels of XIAP, c-IAP2, and survivin in MC3T3-E1 cells under iron overload decreased, which promoted cell apoptosis.

In recent years, studies have shown that miR-1 regulates apoptosis of nitric oxide treated MC3T3-E1 cells by targeting HSP-70, and miR-335-5p inhibits the high glucose induced apoptosis of MC3T3-E1 cells, but the mechanism is not yet clear. The changes in the miRNA expression profile in the apoptosis of FAC-treated MC3T3-E1 cells and the mechanisms by which miRNAs regulate apoptosis of iron-overloaded MC3T3-E1 cells have not been studied. In this study, we confirmed the upregulation of miR-3074-5p in the apoptosis of MC3T3-E1 cells under iron overload conditions by next-generation sequencing and qRT-PCR. KEGG pathway analysis showed that miRNAs may regulate the apoptosis of iron-overloaded MC3T3-E1 cells by targeting apoptosis-related mRNAs associated with the FoxO and/or mTOR signaling pathways. Target mRNAs of miR3074-5p were predicted by using bioinformatics analysis. Among the predicted target mRNAs, Smad4 mRNA is related to the FoxO signaling pathway, and its downregulation can promote apoptosis. Previous studies have confirmed that the abrogation of Smad4 in chondrocytes and the blockade of Smad4 in cardiac myocytes result in increased apoptosis [32-34].

Smad4 is a key mediator in the signal transduction of transforming factor $\beta$ (TGF$\beta$ )-Smad. Many previous studies have found that the BMP/Smad signaling pathway is involved in the regulation of osteogenic differentiation of osteoblasts. Inhibition of the expression of Smad1 and Smad4 can restrain the osteogenic differentiation of MC3T3-E1 cells [35, 36]. However, there are few studies on whether Smad4 is involved in the regulation of apoptosis in MC3T3-E1 cells. TGF-beta activates ERK in a Smad4-dependent manner and knockdown of Smad4 may inhibit ERK phosphorylation [37, 38]. ERK phosphorylation can promote the expression of antiapoptotic proteins such as Bcl-2 and inhibit the expression of proapoptotic proteins such as Bad. When ERK phosphorylation was blocked, these apoptosis inhibition effects were lost, and apoptosis was promoted. In addition, inhibition of ERK phosphorylation could cause activation of the FOXO transcription factor, leading to cell apoptosis [39]. It has been reported that activation of Smad4 can inhibit AKT phosphorylation and induce apoptosis of gastric cancer cells [40], but no relevant article has suggested that activation of Smad4 can block phosphorylation of Stat3 and lead to apoptosis. In this study, the expression of Smad4 in MC3T3-E1 cells under iron overload conditions was decreased, which could be reversed by inhibition of miR-3074-5p. Moreover, the expression of Smad4 in MC3T3-E1 cells under iron overload conditions was further reduced via overexpression of miR-3074-5p. These results suggested that 
miR-3074-5p may regulate the degradation of Smad4 mRNA by targeting its 3'UTR, which was confirmed by the results of the dual-luciferase reporter assay.

In addition, it has been reported that iron overload can also inhibit the differentiation of MC3T3-E1 cells by inhibiting ALP activity and the expression of coll mRNA and protein [12], but its potential molecular mechanism and related signaling pathways still need to be further elucidated.

\section{Conclusions}

In conclusion, this study showed that iron overload induced apoptosis in MC3T3-E1 cells, and miR-3074-5p is involved in viability inhibition of iron-overloaded MC3T3E1 cells by directly targeting Smad4 (Fig. 5c). Further clinical analysis of miR-30745 p-Smad4 in patients with osteoporosis should demonstrate the clinical relevance of this study, providing a new idea for solving the problem of bone loss in osteoporosis patients.

\section{Abbreviations}

AKT: Serine/threonine kinase; C-IAP2: Cellular inhibitor of apoptosis protein-2; ERK: Extracellular regulated MAP kinase; FAC: Ferric ammonium citrate; FoxO: Forkhead box O; miRNA: MicroRNA; mTOR: Mechanistic target of rapamycin kinase; MUT: Mutant; p-AKT: Phosphorylated AKT; p-ERK: Phosphorylated ERK; p-Stat3: Phosphorylated Stat3; qRT-PCR: Real-time quantitative reverse transcription PCR; Smad4: SMAD family member 4; Stat3: Signal transducer and activator of transcription 3; UTR: Untranslated region; WT: Wild type; XIAP: X-linked inhibitor of apoptosis.

\section{Supplementary Information}

The online version contains supplementary material available at https://doi.org/10.1186/s11658-021-00281-w.

Additional file 1 . Results of bioinformatics analysis.

Acknowledgements

This study was supported by the Department of Biochemistry, Medical College, Jinan University.

Authors' contributions

YF and PYH performed the experiments and collected data; YF wrote the manuscript; JWJ and SSL provided suggestions and comments on project and manuscript preparation; WDK and WZ participated in project design and provided funds. WJC, PLW and CL participated in manuscript preparation and revision. All authors read and approved the final manuscript.

\section{Funding}

This study was supported by the Science and Technology Planning Project of Guangdong Province, China [Grant nos. 2015A020214015 and 2016A020220015] and the Science and Technology Planning Project of Guangzhou, China [Grant no. 201904010145].

\section{Availability of data and materials}

All data generated or analyzed during this study are included in this manuscript.

\section{Declarations}

Ethics approval and consent to participate

Not applicable.

\section{Consent for publication}

Not applicable.

Competing interests

The authors declare that they have no competing interests.

\section{Author details}

${ }^{1}$ Department of Orthodontics, The First Affiliated Hospital of Jinan University, No.613 Huangpu Road West, Guangzhou 510630, China. ${ }^{2}$ Department of Biochemistry, Basic Medical College, Jinan University, No.601 Huangpu Road West, Guangzhou 510632, China. ${ }^{3}$ Department of Orthodontics, School of Stomatology, Jinan University, Guangzhou, China. 
Received: 22 July 2021 Accepted: 10 August 2021

Published online: 16 August 2021

\section{References}

1. Liu G, Men P, Kenner GH, Miller SC. Age-associated Iron accumulation in bone: implications for postmenopausal osteoporosis and a new target for prevention and treatment by chelation. Biometals. 2006;19(3):245-51.

2. Burge R, Dawson-Hughes B, Solomon DH, Wong JB, King A, Tosteson A. Incidence and economic burden of osteoporosis-related fractures in the United States, 2005-2025. J Bone Miner Res. 2007;22(3):465-75.

3. Chan YL, Pang LM, Chik KW, Cheng JC, Li CK. Patterns of bone diseases in transfusion-dependent homozygous thalassaemia major: predominance of osteoporosis and desferrioxamine-induced bone dysplasia. Pediatr Radiol. 2002;32(7):492-7.

4. Vogiatzi MG, Autio KA, Schneider R, Giardina PJ. Low bone mass in prepubertal children with thalassemia major: insights into the pathogenesis of low bone mass in thalassemia. J Pediatr Endocrinol Metab. 2004;17(10):1415-21.

5. Xu Z, Sun W, Li Y, Ling S, Zhao C, Zhong G, Zhao D, Song J, Song H, Li J, You L, Nie G, Chang Y, Li Y. The regulation of iron metabolism by hepcidin contributes to unloading-induced bone loss. Bone. 2017;94:152-61.

6. Schnitzler CM, Schnaid E, MacPhail AP, Mesquita JM, Robson HJ. Ascorbic acid deficiency, iron overload and alcohol abuse underlie the serve osteoporosis in black African patients with hip fractures - a bone histomorphometric study. Calcif Tissue Int. 2005;76(2):79-89.

7. Isomura H, Fujie K, Shibata K, Inoue N, lizuka T, Takebe G, Takahashi K, Nishihira J, Izumi H, Sakamoto W. Bone metabolism and oxidative stress in postmenopausal rats with iron overload. Toxicology. 2004;197(2):93-100.

8. Jeney V. Clinical impact and cellular mechanisms of iron overload-associated bone loss. Front Pharmacol. 2017:8:77.

9. Tsay J, Yang Z, Ross FP, Cunningham-Rundles S, Lin H, Coleman R, Mayer-Kuckuk P, Doty SB, Grady RW, Giardina PJ, Boskey AL, Vogiatzi MG. Bone loss caused by iron overload in a murine model: importance of oxidative stress. Blood. 2010;116(14):2582-9.

10. Ke JY, Cen WJ, Zhou XZ, Li YR, Kong WD, Jiang JW. Iron overload induces apoptosis of murine preosteoblast cells via ROS and inhibition of AKT pathway. Oral Dis. 2017;23(6):784-94.

11. Tian Q, Wu S, Dai Z, Yang J, Zheng J, Zheng Q, Liu Y. Iron overload induced death of osteoblasts in vitro: involvement of the mitochondrial apoptotic pathway. Peer J. 2016;4:e2611.

12. Yamasaki K, Hagiwara H. Excess iron inhibits osteoblast metabolism. Toxicol Lett. 2009;191(2-3):211-5.

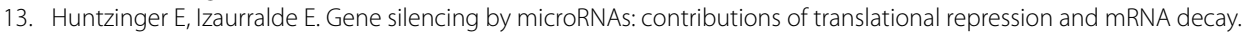
Nat Rev Genet. 2011;12(2):99-110.

14. Krol J, Loedige I, Filipowicz W. The widespread regulation of microRNA biogenesis, function and decay. Nat Rev Genet. 2010;11(9):597-610.

15. Li Z, Rana TM. Therapeutic targeting of microRNAs: current status and future challenges. Nat Rev Drug Discov. 2014;13(8):622-38.

16. MicroRNAs GP. MicroRNAs: recently discovered key regulators of proliferation and apoptosis in animal cells: Identification of miRNAs regulating growth and survival. Cytotechnology. 2007;53(1-3):55-63.

17. Subramanian S, Steer CJ. MicroRNAs as gatekeepers of apoptosis. J Cell Physiol. 2010;223(2):289-98.

18. Lynam-Lennon N, Maher SG, Reynolds JV. The roles of microRNA in cancer and apoptosis. Biol Rev. 2009;84(1):55-71.

19. Lee YE, Hong CY, Lin YL, Chen RM. MicroRNA-1 participates in nitric oxide-induced apoptotic insults to MC3T3-E1 cells by targeting heat-shock protein-70. Int J Biol Sci. 2015;11(3):246-55.

20. Lu XZ, Yang ZH, Zhang HJ, Zhu LL, Mao XL, Yuan Y. MiR-214 protects MC3T3-E1 osteoblasts against $\mathrm{H}_{2} \mathrm{O}_{2}$-induced apoptosis by suppressing oxidative stress and targeting ATF4. Eur Rev Med Pharmacol Sci. 2017;21(21):4762-70.

21. Yin N, Zhu L, Ding L, Yuan J, Du L, Pan M, Xue F, Xiao H. MiR-135-5p promotes osteoblast differentiation by targeting HIF1AN in MC3T3-E1 cells. Cell Mol Biol Lett. 2019;24:51.

22. Li H, Tian X, Wang P, Huang M, Xu R, Nie T. MicroRNA-582-3p negatively regulates cell proliferation and cell cycle progression in acute myeloid leukemia by targeting cyclin B2. Cell Mol Biol Lett. 2019;24:66.

23. Zhang $X, G e Y L$, Zhang SP, Yan P, Tian RH. Downregulation of KDR expression induces apoptosis in breast cancer cells. Cell Mol Biol Lett. 2014;19(4):527-41.

24. Sun Y, Wang C, Wang L, Dai Z, Yang K. Arsenic trioxide induces apoptosis and the formation of reactive oxygen species in rat glioma cells. Cell Mol Biol Lett. 2018;23:13.

25. Cen WJ, Feng Y, Li SS, Huang LW, Zhang T, Zhang W, Kong WD, Jiang JW. Iron overload induces G1 phase arrest and autophagy in murine preosteoblast cells. J Cell Physiol. 2018;233(9):6779-89.

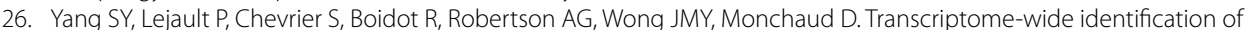
transient RNA G-quadruplexes in human cells. Nat Commun. 2018;9(1):4730.

27. Budihardjo I, Oliver H, Lutter M, Luo X, Wang X. Biochemical pathways of caspase activation during apoptosis. Annu Rev Cell Dev Biol. 1999;15:269-90.

28. Zhang B, Yin CP, Zhao Q, Yue SW. Upregulation of HIF-1 a by hypoxia protect neuroblastoma cells from apoptosis by promoting survivin expression. Asian Pac J Cancer Prev. 2014;15(19):8251-7.

29. Deng S, Dai G, Chen S, Nie Z, Zhou J, Fang H, Peng H. Dexamethasone induces osteoblast apoptosis through ROSPI3K/AKT/GSK3beta signaling pathway. Biomed Pharmacother. 2019;110(19):602-8.

30. Wang H, Xia W, Long G, Pei Z, Li Y, Wu M, Wang Q, Zhang Y, Jia Z, Chen H. Isoquercitrin ameliorates cisplatin-induced nephrotoxicity via the inhibition of apoptosis, inflammation, and oxidative stress. Front Pharmacol. 2020;11:599416.

31. Wang R, Zhang D, Sun K, Peng J, Zhu W, Yin S, Tang D, Wu Y. Amygdalin promotes the activity of T cells to suppress the progression of HBV-related hepatocellular carcinoma via the JAK2/STAT3 signaling pathway. BMC Infect Dis. 2021;21(1):56

32. Zhang J, Tan X, Li W, Wang Y, Wang J, Cheng X, Yang X. Smad4 is required for the normal organization of the cartilage growth plate. Dev Biol. 2005;284(2):311-22. 
33. Li Y, Du Y, Cao J, Gao Q, Li H, Chen Y, Lu N. MiR-130a inhibition protects rat cardiac myocytes from hypoxia-triggered apoptosis by targeting Smad4. Kardiol Pol. 2018;76(6):993-1001.

34. Simeone DM, Zhang L, Graziano K, Nicke B, Pham T, Schaefer C, Logsdon CD. Smad4 mediates activation of mitogen-activated protein kinases by TGF-beta in pancreatic acinar cells. Am J Physiol Cell Physiol. 2001;281 (1):311-9.

35. Kato RB, Roy B, De Oliveira FS, Ferraz EP, De Oliveira PT, Kemper AG, Hassan MQ, Rosa AL, Beloti MM. Nanotopography directs mesenchymal stem cells to osteoblast lineage through regulation of microRNA-SMAD-BMP-2 circuit. J Cell Physiol. 2014;229(11):1690-6.

36. Kim DY, Park YG, Quan HY, Kim SJ, Jung MS, Chung SH. Ginsenoside Rd stimulates the differentiation and mineralization of osteoblastic MC3T3-E1 cells by activating AMP-activated protein kinase via the BMP-2 signaling pathway. Fitoterapia. 2012;83(1):215-22.

37. Li J, Huang J, Dai L, Yu D, Chen Q, Zhang X, Dai K. miR-146a, an IL-1 $\beta$ responsive miRNA, induces vascular endothelial growth factor and chondrocyte apoptosis by targeting Smad4. Arthritis Res Ther. 2012;14(2):R75.

38. Balmanno K, Cook SJ. Tumor cell survival signaling by the ERK1/2 pathway. Cell Death Differ. 2009;16(3):368-77.

39. Roy SK, Srivastava RK, Shankar S. Inhibition of PI3K/AKT and MAPK/ERK pathways causes activation of FOXO transcription factor, leading to cell cycle arrest and apoptosis in pancreatic cancer. J Mol Signal. 2010;5:10.

40. Kundu J, Wahab SM, Kundu JK, Choi YL, Erkin OC, Lee HS, Park SG, Shin YK. Tob1 induces apoptosis and inhibits proliferation, migration and invasion of gastric cancer cells by activating Smad4 and inhibiting beta-catenin signaling. Int J Oncol. 2012:41(3):839-48.

\section{Publisher's Note}

Springer Nature remains neutral with regard to jurisdictional claims in published maps and institutional affiliations.

- fast, convenient online submission

- thorough peer review by experienced researchers in your field

- rapid publication on acceptance

- support for research data, including large and complex data types

- gold Open Access which fosters wider collaboration and increased citations

- maximum visibility for your research: over $100 \mathrm{M}$ website views per year

At BMC, research is always in progress.

Learn more biomedcentral.com/submissions 